\title{
Spectral generalization testing with pigeons using brief discrete trials ${ }^{1}$
}

\author{
MORRIS K. HOLLAND AND WILLIAM M. BAKER
}

DUKE UNIVERSITY

Spectral generalization gradients were obtained from pigeons with a brief discrete trial procedure and examined at different stages of extinction. To reduce the dependence of response probability on elapsed time in the test, interpolated noncontingent feedings were given at intervals throughout four days of testing. While responding extinguished prior to the "free" feedings, generalization gradients reappeared immediately thereafter. Extent of generalization appeared to be a function of momentary response probability.

The procedure of Guttman \& Kalish (1956) for obtaining generalization gradients in extinction following variable interval (VI) training to a single stimulus value has proved a valuable tool for the analysis of behavior. The length of the VI trial, however, limits the usefulness of this procedure for certain types of questions. An alternative generalization testing procedure, explored in the present study, involves short, closely spaced discrete trials, each trial terminated by a single peck, and employs a probability measure of response. The relatively brief sampling time of the discrete trial procedure permits a more detailed tracking of the behavioral change during the generalization test. The present study was designed to examine the change in extent of generalization during extinction and recovery of responding.

Method

Twelve White Carneaux pigeons, maintained at $80 \%$ of their ad lib weights, were trained to peck a Plexiglas key illuminated from behind by $550 \mathrm{~m} \mu$ from Bausch and.Lomb monochromators. All control, timing, schedule, and analysis functions were provided by a LINC computer operating on-line. Relays controlled by the LINC activated shutters, magazines, and magazine lights. The LINC in addition sensed switch openings resulting from pecks. During the first 14 days of training percent reinforcement was reduced from $100 \%$ to $10 \%$, and trial time was reduced from 6 to $3 \mathrm{sec}$. For the last six days of training the animals were run on 3 sec trials and $10 \%$ random reinforcement. Throughout training and testing, trials were terminated either when the $3 \mathrm{sec}$ had elapsed or when a peck occurred.

The 11 stimuli used for the generalization test consisted of monochromatic values from 500 to 600 $\mathrm{m} \mu$, at $10 \mathrm{~m} \mu$ intervals. Each sequential block of 11 3 sec trials was composed of the 11 test stimuli presented in random order separated by 2 sec blackouts. Four days of generalization testing occurred, consisting of 4000 trials. Interpolated feedings ("free" feedings) occurred at regular intervals throughout the test. Each free feeding was preceded by a $20 \mathrm{sec}$ blackout; then grain magazines and magazine lights were activated for $10 \mathrm{sec}$, after which testing continued. On the first day of generalization testing (1000 trials), free feeding occurred after 600 and again after 800 trials. On the second day, free feeding occurred every 200 trials. Free feeding occurred every 33 trials on the third day and every 66 trials on the fourth day of testing.

\section{Results and Discussion}

The effect of free feeding on recovery of responding in pigeons has previously been described by Reid (1958). Figure 1 shows this effect in the present experiment. In this figure, average probability of response is recorded for successive blocks of 33 trials (every three times through the schedule of 11 stimuli) for the first two days of generalization testing. By the end of the initial run of 600 trials (A), responding had stopped. At this time, following a 20 sec blackout, a $10 \mathrm{sec}$ feeding was given and responding was recovered. After 200 additional trials (B), response probability was again close to zero, and a second feeding was given, followed by the responding shown in interval $C$. The second day of testing consisted of 1600 trials with free feedings at the end of every 200 trials. The eight cycles of extinction and recovery on the second day were averaged for the response probability shown in $D$ of Fig. 1. While responding extinguished prior to each free feeding, generalization gradients reappeared in

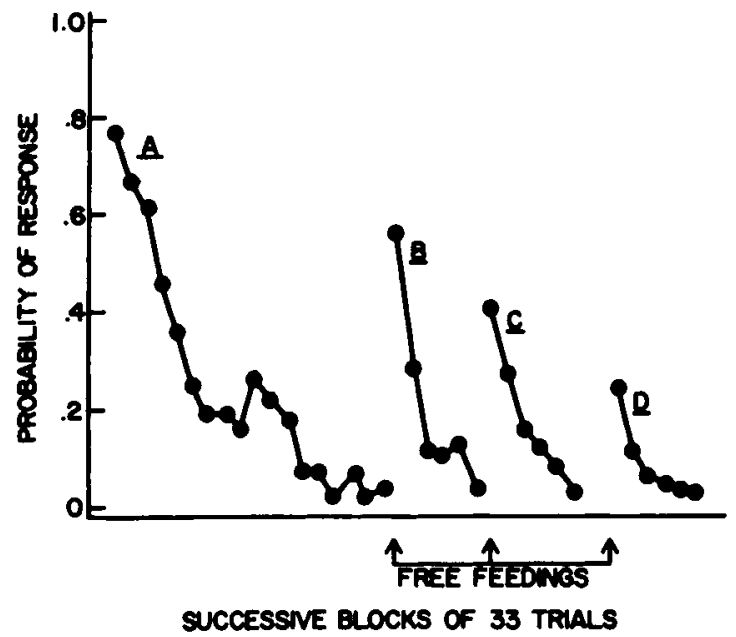

Fig. 1. Average probability of response for successive 33-trial blocks during the first and second days of the generalization test. "Free" feedings occurred before $B$ and $C$ on the first day, and before each of the eight cycles averaged for $D$ on the second day. 


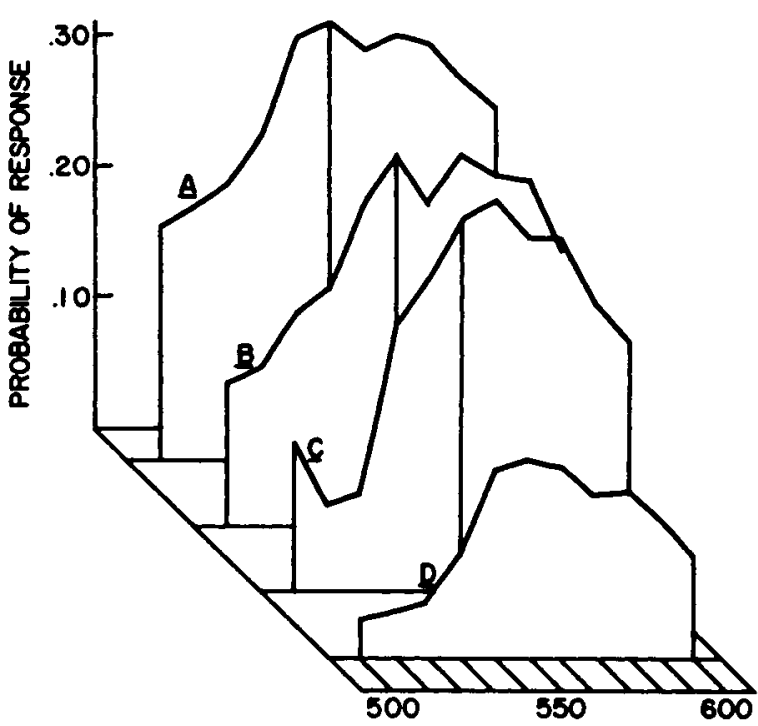

Fig. 2. Generalization gradients produced during the cycles of responding shown in Fig. 1.

the responding which followed the feedings. These "recovered" generalization gradients, corresponding to the cycles shown in Fig. 1, appear in Fig. 2.

It will be noted that the gradients produced with the discrete trials procedure are bowl-shaped and asymmetrical in comparison with the relatively symmetrical "spikes" obtained by a VI schedule and its accompanying rate measure. Hanson \& Guttman (1961) have shown that when rate data gathered under a VI schedule are transformed to a probability measure (1.e., the probability that a bird will peck at least once to a given stimulus presentation), the resulting gradients are asymmetrical, bowl-shaped curves. Clearly, the difference between the gradients comes from the additional pecks during a trial, which are not permitted in the discrete trial procedure.

An advantage of using discrete trials, however, is that all stimuli can be sampled in a relatively short period of time, e.g., $55 \mathrm{sec}$ in the present experiment. In comparison, the typical VI procedure requires 5 to $10 \mathrm{~min}$ to sample each of the stimuli once. Thus, the discrete trial probability measure may be more sensitive to behavioral changes occurring during the generalization test. That rapid changes do occur is shown in Fig. 3, which gives average gradients produced in the first, second, and third minute of testing following the free feedings on Test Days 3 and 4 . After 10 cycles of extinction and recovery on Test Days 1 and 2, responding on Days 3 and 4 declined very rapidly after a free feeding.

The interpolated free feedings produced cyclical

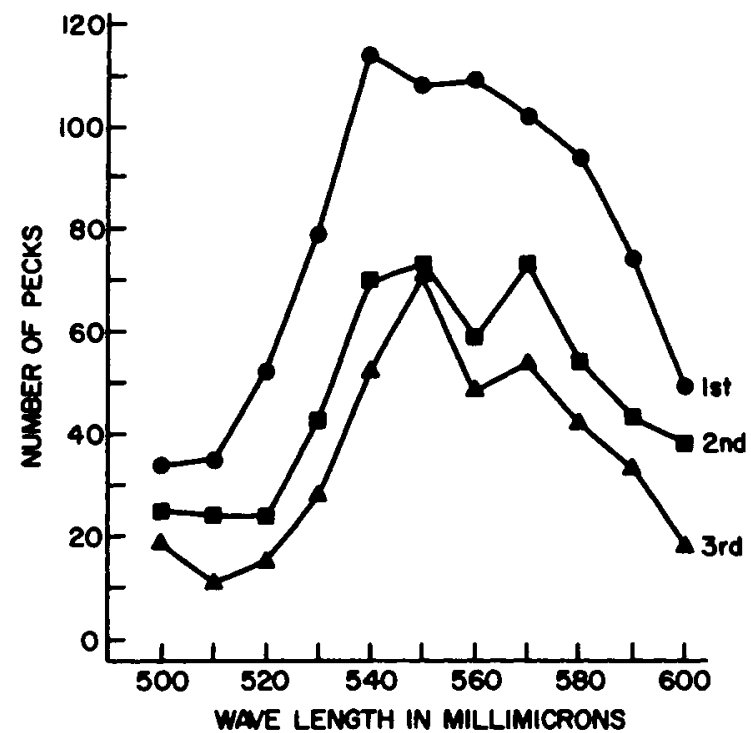

Fig. 3. Gradients produced in the first, second, and third min after "free" feeding on Test Days 3 and 4.

changes not only in response probability but also in extent of generalization. When response probability was low, relatively more responding occurred to the training stimulus. The overall percentage of responses made to the training stimulus $(550 \mathrm{~m} \mu)$ was $13 \%$. During the first $1 \mathrm{~min}$ period following the interpolated feedings on Test Days 3 and 4 (Fig. 3), percentage of responses made to the training stimulus was also $13 \%$. As response probability declined over the $3 \mathrm{~min}$ period shown in Fig. 3, this percentage increased from $13 \%$ to $14 \%$ to $18 \%$. For the four days of testing, when response probability was so low that only one response was made during an 11 trial block in which each stimulus was presented, the percentage of responses made to the training stimulus was $25 \%$. Thus, extent of generalization appeared to be a function of momentary response probability, when response probability was varied cyclically throughout four days of testing.

\section{References}

GUTTMAN, N., \& KALISH, H. I. Discriminability and stimulus generalization. J. exp. Psychol., 1956, 51, 79-88.

HANSON, H. M., \& GUTTMAN, N. The use of a behavioral stimulant in the study of stimulus generalization. J. exp. Anal. Behav., 1961, $4,209-212$.

REID, R. L. The role of the reinforcer as a stimulus. Brit. J. Psychol, $1958,49,202-209$.

Note

1. This research was supported by a NASA training grant to the first author and by grants MH 06661 and MH 08351 from NIH to C. Alan Boneau. 